\section{Statistical validity of interleukin-6 as a biomarker for the diagnosis of early-onset neonatal sepsis}

\author{
Sriparna Basu, ${ }^{1}$ Shashikant Dewangan, ${ }^{1}$ \\ Shampa Anupurva, ${ }^{2}$ Ashok Kumar ${ }^{1}$ \\ 1 Department of Pediatrics and \\ 2Department of Microbiology, Institute \\ of Medical Sciences, Banaras Hindu \\ University, Varanasi, India
}

\section{Abstract}

Use of empirical antibiotics in neonates with risk factors of early-onset neonatal sepsis (EOS) is a common practice. A laboratory parameter is needed to help in the accurate diagnosis of EOS to avoid unnecessary use of antibiotics. The aim of this prospective observational cohort study was to compare the statistical validity of cord blood interleukin-6 (IL6) with conventional sepsis screening as an early diagnostic marker for EOS. Eighty-seven neonates with antenatal risk factors for sepsis were followed up for $72 \mathrm{~h}$ for the development of EOS. Cord blood was collected for measurement of IL-6 concentrations. Blood culture and conventional sepsis screening (total leukocyte count, absolute neutrophil count, C-reactive protein and micro-erythrocyte sedimentation rate) were sent for analysis soon after delivery. The study group comprised of symptomatic neonates with positive blood culture $(n=36)$. An equal number of gestational-age matched asymptomatic neonates without risk factor of sepsis served as controls. Statistical validity of IL-6 was compared with sepsis screening parameters as the diagnostic marker for EOS. Gram negative organisms were the predominant cause of EOS. The most commonly isolated organism was Acinetobacter baumanii. The sensitivity and specificity of IL-6 with a cut-off value of $40.5 \mathrm{pg} / \mathrm{mL}$ and area under curve of 0.959 were 92.3 and $90.48 \%$, respectively. In contrast, the sensitivity and specificity of different parameters of sepsis screening ranged from $37.5-68.75 \%$ and $47.95-57.35 \%$, respectively. In conclusion, cord blood IL- 6 can be used as a highly sensitive and specific early diagnostic marker of EOS at a cut-off concentration of $40.5 \mathrm{pg} / \mathrm{mL}$.

\section{Introduction}

Sepsis is an important cause of neonatal morbidity and mortality in developing coun- tries. ${ }^{1}$ The World Health Organization estimates that 1 million deaths per year are due to neonatal sepsis and $42 \%$ of these deaths occur in the first week of life. ${ }^{2}$ Although a large proportion of neonates are admitted with clinical suspicion of neonatal sepsis, diagnosis is at times difficult because of non-specific laboratory parameters. The practice of starting empirical antimicrobial therapy renders many neonates unduly susceptible to the side effects of antimicrobial agents, increases hospital costs, and promotes the development and spread of resistant bacterial strains. ${ }^{3}$

Blood culture is considered to be the gold standard in the etiological diagnosis of neonatal sepsis but pathogens can actually be identified only in a small subset of patients. Moreover, it takes at least 48-72 $\mathrm{h}$ to obtain a preliminary positive result according to conventional methods. At present, there is no single optimal diagnostic test to identify neonatal sepsis. Hematologic indexes and acute-phase reactants such as C-reactive protein (CRP) remain in widespread use but there are concerns about their reliability which largely emerge from the marked variations in their predictive accuracy. ${ }^{4}$ Therefore, there is the need for a single parameter which can detect neonatal sepsis with reasonable accuracy.

Contrary to late-onset neonatal sepsis (LOS), in which infection is acquired from the surroundings after birth, in EOS, infection is commonly acquired in utero resulting in the development of chorioamnionitis. ${ }^{5}$ Chorioamnionitis activates the fetal immune system leading to fetal inflammatory response syndrome (FIRS). In some neonates, FIRS is amplified and these neonates become symptomatic after birth with clinical manifestations suggestive of EOS. FIRS can be diagnosed by an increased number of proinflammatory cytokines in cord blood, of which interleukin-6 (IL-6) is one of the early mediators released in the circulation. Previous authors have documented high umbilical cord blood IL-6 concentrations in chorioamnionitis. ${ }^{6-8}$ In the present study, we compared the statistical validity of cord blood IL-6 concentrations with conventional sepsis screening as an early diagnostic marker of EOS.

\section{Materials and Methods}

\section{Study population}

The study was conducted in the Neonatal Unit of the Sir Sunderlal Hospital, Banaras Hindu University, India, over a period of one year and was approved by the Institute's Ethics Committee. The initial study group was made up of 87 newborns with one or more risk factors for development of EOS. Neonates were
Correspondence: Sriparna Basu, Department of Pediatrics, Institute of Medical Sciences, Banaras Hindu University, Varanasi, 221005, India.

E-mail: drsriparnabasu@rediffmail.com

Key words: early onset neonatal sepsis, interleukin-6, newborn, sepsis screening.

Contribution: SB designed the study, managed patients and drafted the manuscript. SD helped in record keeping, patient management and drafting of the manuscript. AK helped in patient management and drafting of the manuscript. SA performed the microbiological and pathological tests.

Received for publication: 20 October 2011.

Revision received: 28 November 2011.

Accepted for publication: 8 February 2012.

This work is licensed under a Creative Commons Attribution NonCommercial 3.0 License (CC BYNC 3.0).

(C) Copyright S. Basu et al., 2012

Licensee PAGEPress, Italy

Microbiology Research 2012; 3:e5

doi:10.4081/mr.2012.e5

enrolled into the study, irrespective of their gestational age and sex, after the motherinfant dyads were screened for possible enrolment criteria. Risk factors for development of EOS were defined as:

premature $(<35$ weeks of gestation) or prolonged $(>18 \mathrm{~h}$ ) rupture of membrane; clinical chorioamnionitis defined as intrapartum maternal fever $\left(>37.8^{\circ} \mathrm{C}\right.$ in the period from onset of labor to delivery) with two or more features: fetal tachycardia, uterine tenderness, malodorous vaginal discharge or maternal leukocytosis (leukocytes $\left.>15 \times 10^{9} / \mathrm{L}\right){ }^{9}$

Cord blood was collected for measurement of IL-6 concentrations. Blood culture and conventional sepsis screening (total leukocyte count, absolute neutrophil count, C-reactive protein and micro-erythrocyte sedimentation rate) were sent for analysis soon after delivery. Symptomatic neonates with positive blood culture formed the final study group. An equal number of gestational-age matched asymptomatic neonates without risk factor of sepsis served as controls. Informed and written consent was obtained from the parents of all patients before their inclusion in the study.

Infants with perinatal asphyxia, intrauterine infections (TORCH, congenital syphilis, infective hepatitis, Human Immunodeficiency Virus), other systemic or metabolic diseases, and congenital malformations were excluded. Those who were discharged from hospital before $72 \mathrm{~h}$ of life were also excluded. 


\section{Clinical workup}

Detailed antenatal and intranatal history, including demographic details of the mother and the family, were recorded in all neonates. Thorough clinical examination was made at birth to detect any systemic disease or congenital anomaly. Enrolled subjects were followed up for development of clinical signs of sepsis including: respiratory distress, temperature instability, feeding intolerance/abdominal distension/vomiting, neonatal hyperbilirubinemia, lethargy/irritability, apnea, bradycardia/tachycardia, arterial desaturations, hypotension/poor perfusion, hemorrhagic manifestations, convulsions or abnormal glucose homeostasis (documented by bedside Accuchek dextrostix), recorded every $6 \mathrm{~h}$, for a period of $72 \mathrm{~h}$ after birth. Other investigations, such as laboratory estimation of blood glucose, serum electrolytes, bleeding and coagulation profile, liver and kidney function tests, arterial blood gas analysis, etc., were performed on clinical suspicion as and when indicated. Lumbar puncture and cerebrospinal fluid (CSF) analysis was made on clinical suspicion of meningitis. Neonates were managed according to the protocol of our unit. Progress during hospital stay and outcome were reported.

\section{Collection of samples and laboratory analysis}

After complete delivery, $10 \mathrm{~mL}$ of free flowing cord blood was collected for quantitative estimation of IL-6 in sterilized test tubes from the placental end of the umbilical cord without milking under aseptic conditions. Serum was separated immediately by centrifugation at $3,000 \mathrm{rpm}$ and stored at $-20^{\circ} \mathrm{C}$ until analyzed. IL-6 level was determined by Human IL-6 ELISA kit (Diaclone, GEN-PROBE Inc., San Diego, CA, USA) according to the manufacturer's instructions.

Single-site blood culture was taken within $30 \mathrm{~min}$ of birth from a peripheral vein, prior to starting antibiotics. A $20 \mathrm{~mL}$ blood culture bottle containing sheep brain heart infusion broth was inoculated with $1 \mathrm{~mL}$ of infant's blood under full aseptic conditions. Bacterial growth was noted after $48 \mathrm{~h}$ and then after seven days. Drug sensitivity was determined whenever bacterial growth was noted. Sepsis screening, including total leukocyte count (TLC), absolute neutrophil count (ANC), serum C-reactive protein (CRP), and micro-erythrocyte sedimentation rate ( $\mu$ ESR), was performed in all infants between 12-24 $\mathrm{h}$ after delivery from peripheral venous blood. TLC and ANC were calculated from complete blood count (CBC), carried out on a Wipro diagnostic three chamber CBC machine. Quantitative serum CRP was assessed by nephelometry using the Orion Diagnostica kit, Finland. Micro-ESR was performed in a heparinized capillary tube after maintaining it vertical for $1 \mathrm{~h}$.

\section{Statistical analysis}

The statistical program SPSS version 16.0 (SPSS Inc., Chicago, IL, USA) was used for data analysis. Sensitivity, specificity, positive likelihood ratio (PLR) and negative likelihood ratio (NLR) were calculated at selected cut-off values for IL-6 and sepsis screening parameters. Receiver operating characteristic (ROC) curve analyses with measurement of area under the curve (AUC) were performed to identify the appropriate cut-off values. $\mathrm{P}<0.05$ was considered statistically significant.

\section{Results}

A total of 36 newborns ( 25 males and 11 females) were diagnosed as EOS on the basis of development of clinical signs of sepsis within $72 \mathrm{~h}$ and positive blood culture by conventional methods at $48 \mathrm{~h}$. Baseline parameters of cases and controls were comparable. Mean birth weight of cases and controls was $1632.8 \pm 545$ vs. $1585.2 \pm 632$ g, respectively $(\mathrm{P}>0.05)$ and mean gestational age was

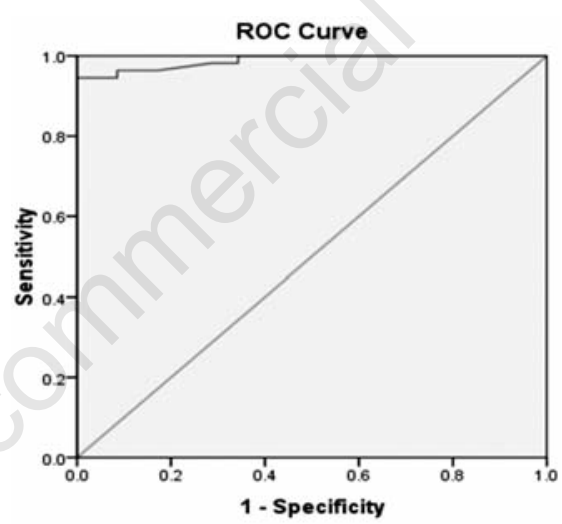

Figure 1. Receiver-operating-characteristic (ROC) curve for interleukin-6. Area under curve 0.959 .

Table 2. Statistical validity indices for interleukin-6 and different parameters of sepsis screen.

\begin{tabular}{lccccc} 
Parameters & Cut off & $\begin{array}{c}\text { Sensitivity } \\
(\%)\end{array}$ & $\begin{array}{c}\text { Specificity } \\
(\%)\end{array}$ & $\begin{array}{c}\text { Positive } \\
\text { likelihood } \\
\text { ratio }\end{array}$ & $\begin{array}{c}\text { Negative } \\
\text { likelihood } \\
\text { ratio }\end{array}$ \\
Interleukin- $6(\mathrm{pg} / \mathrm{mL})$ & $\geq 40.5^{*}$ & 92.3 & 90.48 & 9.69 & 0.08 \\
$\mathrm{TLC}\left(\times 10^{9} / \mathrm{L}\right)$ & $\leq 5$ & 55.5 & 56.2 & 1.27 & 0.79 \\
\hline $\mathrm{ANC}\left(\times 10^{9} / \mathrm{L}\right)$ & $\leq 1.8$ & 37.5 & 49.3 & 1.36 & 1.2 \\
$\mathrm{CRP}(\mathrm{mg} / \mathrm{L})$ & $\geq 10$ & 68.75 & 57.35 & 1.61 & 0.96 \\
\hline$\mu$ ESR $\left(\mathrm{mm}\right.$ in $1^{\text {st }}$ hour $)$ & $\geq 0$ & 66.67 & 47.95 & 1.28 & 1.82 \\
Sepsis screen positive for & - & 80 & 49.3 & 1.58 & 0.41 \\
any 2 parameters & & & & &
\end{tabular}

"Cut-off value of 40.5 for IL-6 was determined by using ROC curve analysis. TLC, total leukocyte count; ANC, absolute neutrophil count; CRP, serum C-reactive protein; $\mu \mathrm{ESR}$, micro-erythrocyte sedimentation rate.

$33.4 \pm 2.8$ vs. $32.8 \pm 2.5$ weeks, respectively $(\mathrm{P}>0.05)$. There was no difference between the groups regarding maternal age, gravidity and parity, nature of antenatal care taken, obstetric complications, and mode of delivery. Both the groups showed male preponderance, none of them was intra-uterine growth restricted, and none of the babies required delivery room resuscitation.

Organisms grown in blood culture are summarized in Table 1. Gram negative organisms were predominant $(91.7 \%)$. The most commonly isolated organism was Acinetobacter baumanii (27.8\%), followed by Klebsiella pneumoniae (22.2\%) and Pseudomonas aeruginosa (19.4\%). Gram positive organisms (Staphylococci) were isolated in only 3 cases (8.3\%).

Validity indexes of IL-6 and sepsis screening parameters are listed in Table 2. Cut-off values for the parameters of positive sepsis screening were defined as: TLC $\leq 5 \times 10^{9} / \mathrm{L}, \mathrm{ANC} \leq 1.8 \times 10^{9} / \mathrm{L}$, $\mathrm{CRP} \geq 10 \mathrm{mg} / \mathrm{L}$, and micro-ESR $\geq 10 \mathrm{~mm}$ in the first hour according to the protocol of our unit. The cut-off value of 40.5 for IL- 6 was determined using ROC curve analysis (Figure 1). Area under curve of IL-6 was 0.959 . Sensitivity, specificity, PLR and NLR of IL-6 at the cut-off value of $40.5 \mathrm{pg} / \mathrm{mL}$ were $92.3 \%, 90.48 \%, 9.69$ and 0.08 , respectively. Sensitivity, specificity, PLR and NLR of other parameters of sepsis screening is shown in Table 2 . Sensitivity ranged individually from 37.5 to $68.75 \%$, specificity from 47.95 to $57.35 \%$, PLR from 1.61 to 1.27 , and NLR from

Table 1. Organisms grown in blood culture in sepsis group $(\mathrm{n}=36)$.

\begin{tabular}{lc} 
Organism & N. isolated (\%) \\
Acinetobacter baumanni & $10(27.8)$ \\
Klebsiella pneumoniae & $8(22.2)$ \\
\hline Pseudomonas aeruginosa & $7(19.4)$ \\
Escherichia coli & $5(13.9)$ \\
\hline Citrobacter & $3(8.3)$ \\
Staphylococcus aureus & $2(5.6)$ \\
\hline Staphylococcus epidermidis & $1(2.8)$ \\
\hline
\end{tabular}


1.82 to 0.79 . Individually, CRP had the highest predictive accuracy among the sepsis screening parameters (sensitivity 68.75\%, specificity 57.35\%, PLR 1.61, and NLR 0.96). ANC showed minimum predictive accuracy (sensitivity $37.5 \%$, specificity $49.3 \%$, PLR 1.36 , and NLR1.2). When these two parameters were combined, sensitivity, specificity, PLR and NLR were $80 \%, 49.3 \%, 1.58$ and 0.41 , respectively.

\section{Discussion}

In this study, we compared the statistical validity of IL-6 with conventional sepsis screening parameters as an early diagnostic marker of EOS. We only included those cases of EOS with positive blood culture to avoid any problems in diagnosis. Males were predominant, and the majority of our study neonates were preterm and low birth weight. Predominant causes of EOS were Gram negative organisms except for 3 cases of Staphylococcal sepsis. The most commonly isolated organism was Acinetobacter (27.8\%) followed by Klebsiella (22.2\%) and Pseudomonas (19.4\%). Two recent reports from eastern and southern India reported Klebsiella to be the most common organism grown in blood culture (48 of 131 and 29 of 39, respectively). ${ }^{10,11}$

We found elevated umbilical cord blood IL-6 concentrations to be a better diagnostic tool compared to conventional sepsis screening in neonates who subsequently developed blood culture positive EOS. IL-6 is an inflammatory cytokine produced by a variety of cells, such as granulocytes, monocytes, lymphocytes, endothelial and some epithelial cell types, in response to bacterial infection. A wide variety of inflammatory mediators induce the release of IL-6 which include tumor necrosis factor alpha, IL-1, endotoxins, etc. ${ }^{12}$ IL-6 is crucial for the initiation of an innate immune response and propagation of acquired immune responses through induction of T-cell activation and proliferation, as well as B-cell differentiation. It stimulates bone marrow and coagulation cascade, and furthermore, as a potent pyrogen, it stimulates the synthesis of a wide spectrum of acute phase proteins in human hepatocytes. ${ }^{12}$ Jarvis et al. have demonstrated higher concentrations of IL-6 in umbilical cord blood at birth in the presence of chorioamnionitis and funisitis as the hallmark of FIRS..$^{13}$ Several studies have demonstrated an early rise in IL6 in neonatal sepsis. ${ }^{14,15}$ IL-6 has been shown to be rapidly released in response to infection, with peak serum levels within $2 \mathrm{~h}$, often before the onset of clinical symptoms and before routine laboratory tests, such as measurement of high-sensitivity CRP (hsCRP), result positive ${ }^{16}$
We recorded IL-6 sensitivity and specificity of 92.3 and $90.48 \%$, respectively, with a cut-off value of $40.5 \mathrm{pg} / \mathrm{mL}$. Different authors have used different cut-off values of IL-6 for the diagnosis of EOS. Nishimaki et al. ${ }^{8}$ have used a cut-off of $54.7 \mathrm{pg} / \mathrm{mL}$. Values as low as 10 $\mathrm{pg} / \mathrm{mL}$ have been used by Martínez Nadal et al. ${ }^{17}$ whereas Smulian et al. ${ }^{18}$ have used a cutoff of $25 \mathrm{pg} / \mathrm{mL}$. A correlation between the severity of acute chorioamnionitis and umbilical venous IL-6 concentrations has also been documented by several authors. Kashlan et al. found that significantly elevated IL-6 concentrations with moderate to severe acute chorioamnionitis, acute deciduitis, and chorionic plate vasculitis. Clinically suspected or proven neonatal sepsis correlated with IL-6 elevations; a $30 \mathrm{pg} / \mathrm{mL}$ cut-off gave $95 \%$ sensitivity and $78 \%$ specificity, and a $100 \mathrm{pg} / \mathrm{mL}$ cutoff resulted in $84 \%$ sensitivity and $91 \%$ specificity- ${ }^{19}$ On the other hand, Khassawneh et al. found the cut-off value of $18.2 \mathrm{pg} / \mathrm{mL}$ to be associated with $87 \%$ sensitivity and $50 \%$ specificity. ${ }^{20}$ In the study carried out by Schefold et al, the sensitivity and specificity of IL- 6 was found to be 82.6 and $86.5 \%$, respectively. ${ }^{16}$

Hematologic markers which increase in response to infection include microscopic examination of the blood for white blood cells: total and differential leukocyte count, absolute neutrophil count, and immature to total neutrophil (I:T) ratio. Different authors found significant heterogeneity in the predictive value of hematologic indexes of sepsis screen. The possible sources of heterogeneity were: population, age, whether the subjects were at term or preterm, methodological quality, different leukocyte indexes, different cut-off values, and interpretation of test results by different laboratory observers. The majority of the studies have demonstrated low detection sensitivity for neonatal infection. Even the combination of all factors failed to reach an appropriate sensitivity and specificity in EOS. ${ }^{21-23}$

The most commonly used acute phase reactant is CRP which has been widely studied as a diagnostic tool in neonatal sepsis and also as an indicator of response to therapy. However, CRP takes 12-24 h to increase to measurable levels; it has a very long half-life and it takes 5 7 days to normalize after eradication of the infectious agent. ${ }^{24}$ Several authors have reported that CRP can not be used as a good predictor of neonatal sepsis alone. ${ }^{25-27}$

Sepsis screening did not perform well in our study with sensitivity of $80 \%$ and NLR of 0.41 . Kudawla et al. reported a sensitivity of $48.3 \%$, specificity of 70\%, PLR of 1.61 and NLR of $0.738 .{ }^{28}$ We have taken a relatively higher cutoff level of CRP (10 mg/L) to diagnose EOS. The study of Khassawneh et al. showed that a CRP of over $4 \mathrm{mg} / \mathrm{L}$ could give the best cut-off value with $95.7 \%$ sensitivity, $88.9 \%$ specificity,
78.6\% PPV and 98\% NPV. ${ }^{20}$ The authors concluded that this CRP cut-off value offered the best single available test to predict or exclude Gram negative neonatal infections.

However, this study was not without limitations. Sample size was small because we strictly included only blood culture positive cases. The blood culture positivity rate in our unit has remained $30-35 \%$ over the last five years. We could not measure I:T ratio because we did not have the necessary facilities. Moreover, because of financial constraints, we could not measure multiple inflammatory cytokines to increase the specificity of the diagnostic tests, and we could not make serial measurements of IL-6 concentrations, although IL-6 kinetics could have been a better parameter to use to assess its diagnostic utility.

In conclusion, Gram negative organisms were the predominant cause of EOS in our study. Interleukin-6 concentrations in umbilical cord blood are elevated in chorioamnionitis. Umbilical cord blood IL-6 concentrations were found to be a highly sensitive and specific early diagnostic parameter at a cut-off concentration of $40.5 \mathrm{pg} / \mathrm{mL}$ in preterm, low birth weight neonates with EOS. Conventional sepsis screening markers, including hematologic indices and C-reactive protein, are not reliable early markers for early-onset neonatal sepsis.

\section{References}

1. Maayan-Metzger A, Barzilai A, Keller N, Kuint J. Are the "good old" antibiotics still appropriate for early-onset neonatal sepsis? A 10 year survey. Isr Med Assoc J 2009;11: $138-42$.

2. Lawn JE, Cousens S, Zupan J. 4 million neonatal deaths: when? Where? Why? Lancet 2005;365:891-900.

3. Murray BE. Can antibiotic resistance be controlled? N Engl J Med 1994;330:1229-30.

4. Malik A, Hui CP, Pennie RA, Kirpalani H. Beyond the complete blood cell count and Creactive protein: a systematic review of modern diagnostic tests for neonatal sepsis. Arch Pediatr Adolesc Med 2003;157:5116.

5. Toti P, De Felice C, Occhini R, et al. Spleen Depletion in Neonatal Sepsis and Chorioamnionitis. Am J Clin Pathol 2004;122:76571.

6. Yoon BH, Romero R, Park JS, et al. The relationship among inflammatory lesions of the umbilical cord (funisitis), umbilical cord plasma interleukin 6 concentration, amniotic fluid infection, and neonatal sepsis. Am J Obstet Gynecol 2000;183:1124-9.

7. Martin H, Olander B, Norman M. Reactive hyperemia and interleukin 6 , interleukin 8 , and tumor necrosis factor- $\alpha$ in the diagno- 
sis of early-onset neonatal sepsis. Pediatrics 2001;108:1-6.

8. Nishimaki S, Sato M, An H, et al. Comparison of markers for fetal inflammatory response syndrome: fetal blood interleukin6 and neonatal urinary beta(2)-microglobulin. J Obstet Gynaecol Res 2009;35:472-6.

9. Alexander JM, Gilstrap LC, Cox SM, et al. Clinical chorioamnionitis and the prognosis for very low birth weight infants. Obstet Gynecol 1998;91:725-9.

10. Viswanathan R, Singh AK, Mukherjee S, et al. Aetiology and antimicrobial resistance of neonatal sepsis at a tertiary care centre in eastern India: a 3 year study. Indian $\mathrm{J}$ Pediatr 2011;78:409-12.

11. Zakariya BP, Bhat V, Harish BN, et al. Neonatal sepsis in a tertiary care hospital in south India: Bacteriological profile and antibiotic sensitivity pattern. Indian $\mathrm{J}$ Pediatr 2011;78:413-7.

12. Rogers BB, Alexander JM, Head J, et al. Umbilical Vein Interleukin-6 Levels Correlate With the Severity of Placental Inflammation and Gestational Age. Hum Pathol 2002;33:335-40.

13. Jarvis JN, Deng L, Berry SM, et al. Fetal cytokine expression in utero detected by reverse transcriptase polymerase chain reaction. Pediatr Res 1995;37:450-4.

14. Martin H, Olander B, Norman M. Reactive hyperemia and interleukin 6 , interleukin 8 , and tumor necrosis factor- $\alpha$ in the diagno- sis of early-onset neonatal sepsis. Pediatrics 2001;108:1-6.

15. Kantar M, Kültürsay N, Kütükçüler N, et al. Plasma concentrations of granulocytemacrophage colony-stimulating factor and interleukin-6 in septic and healthy preterms. Eur J Pediatr 2000;159:156-7.

16. Schefold JC, Hasper D, von Haehlin S, et al. Interleukin-6 serum level assessment using a new qualitative point-of-care test in sepsis. A comparison with ELISA measurements. Clinical Biochem 2008;41:893-8.

17. Martínez Nadal S, Elizari Saco MJ, Fernández Delclos D, et al. Cord blood levels of interleukin 6 in preterm infants as an early marker of neonatal morbidity. An Pediatr 2008;68:218-23.

18. Smulian JC, Vintzileos AM, Lai YL, et al. Maternal chorioamnionitis and umbilical vein interleukin-6 levels for identifying early neonatal sepsis. J Matern Fetal Med 1999;8:88-94.

19. Kashlan F, Smulian J, Shen-Schwarz S, et al. Umbilical vein interleukin 6 and tumor necrosis factor alpha plasma concentrations in the very preterm infant. Pediatr Infect Dis J 2000;19:238-43.

20. Khassawneh M, Hayajneh WA, Kofahi H, et al. Diagnostic Markers for Neonatal Sepsis: Comparing C-reactive Protein, Interleukin6 and Immunoglobulin M. Scand J Immunol 2007;65;171-5.

21. Kite P, Millar MR, Gorham P, Congdon P.
Comparison of five tests used in diagnosis of neonatal bacteraemia. Arch Dis Childhood 1988; 63:639-43.

22. Manroe BL, Weinberg AG, Rosenfeld CR, Browne R. The neonatal blood count in health and disease. I. Reference values for neutrophilic cells. J Pediatr 1979;95:89-98.

23. Zipursky A, Palko J, Milner R, Akenzua GI. The hematology of bacterial infections in premature infants. Pediatrics 1976;57:83953.

24. Mathers NJ, Pohlandt F. Diagnostic audit of C-reactive protein in neonatal infection. Eur J Pediatr 1987;146:147-51.

25. Sann L, Bienvenu F, Bienvenu J. Evolution of serum prealbumin, C-reactive protein, and orosomucoid in neonates with bacterial infection. J Pediatr1984;105:977-81.

26. Povoa P, Coelho L, Almeida E, et al. C-reactive protein as a marker of infection in critically ill patients. Clin Microbiol Infect 2005;11:101-8.

27. Schmit X, Vincent JL. The time course of blood C-reactive protein concentrations in relation to the response to initial antimicrobial therapy in patients with sepsis. Infection 2008;36:213-9.

28. Kudawla M, Dutta S, Narang A. Validation of a clinical score for the diagnosis of late onset neonatal septicemia in babies weighing 1000-2500 g. J Trop Pediatr 2008; 54:66-9. 